Draft VERSiOn June 7, 2021

Preprint typeset using $\mathrm{LATEX}_{\mathrm{E}}$ style emulateapj v. 12/16/11

\title{
DYNAMICAL PROPERTIES OF INTERNAL SHOCKS REVISITED
}

\author{
Asaf Pe'er ${ }^{1}$, Killian Long ${ }^{1}$, and Piergiorgio Casella ${ }^{2}$ \\ Draft version June 7, 2021
}

\begin{abstract}
Internal shocks between propagating plasma shells, originally ejected at different times with different velocities are believed to play a major role in dissipating the kinetic energy, thereby explaining the observed lightcurve and spectra in a large range of transient objects. Even if initially the colliding plasmas are cold, following the first collision the plasma shells are substantially heated, implying that in a scenario of multiple collisions, most collisions take place between plasmas of non-zero temperatures. Here, we calculate the dynamical properties of plasmas resulting from a collision between arbitrarily hot plasma shells, moving at arbitrary speeds. We provide simple analytical expressions valid for both the ultra-relativistic and Newtonian velocities, for both hot and cold plasmas. We derive the minimum criteria required for the formation of the two-shock wave system, and show that in the relativistic limit, the minimum Lorentz factor is proportional to the square root of the ratio of the initial plasmas enthalpies. We provide basic scaling laws of synchrotron emission from both the forward and reverse shock waves, and show how these can be used to deduce the properties of the colliding shells. Finally, we discuss the implications of these results in the study of several astronomical transients, such as $\mathrm{x}$-ray binaries, radio loud quasars and gamma-ray bursts.
\end{abstract}

\section{INTRODUCTION}

Lightcurves of many astronomical transients that are characterized by strong outflows (jets) show substantial variability, observed on timescales as fast as millisecond and possibly even faster. Several examples include blazars (Marscher 1980), gamma-ray bursts (GRBs; Norris et al. 1996) and x-ray binaries (XRBs; Fender 2001). A leading model proposed to explain these variable lightcurves is the internal shocks model. The basic idea is that variability within the inner engine results in fluctuations in the ejection of plasmas. Thus, the ejected material propagates as a collection of "plasma shells". Each individual shell is accelerated and then propagates at some terminal velocity which is independent on the terminal velocities of the other plasma shells. At a second stage, shells that were ejected at later times but with faster speeds, catch up with the slower shells ahead. The collision between the plasma shells results in the formation of two shock waves (forward and reverse) that propagate, respectively, into the slow and fast shells. These shock waves dissipate part of the shells' kinetic energy, which is then radiated away. Following the collision, the colliding shells are assumed to merge and continue propagating together (i.e., the collision is considered as a plastic collision), and are therefore subject to a subsequent collision with a third incoming, faster shell. This scenario of multiple collisions therefore results in the observed variable lightcurve.

Such models were proposed to explain the knots in active galactic nuclei [AGN] jets (Rees 1978). They have been in wide use since the 1990's in explaining the rapid variability observed during the prompt phase of many GRBs (e.g., Rees \& Meszaros 1994; Fenimore et al. 1996; Sari \& Piran 1997; Kobayashi et al. 1997; Daigne \& Mochkovitch 1998;

\footnotetext{
${ }^{1}$ Physics Department, University College Cork, Cork, Ireland

2 INAF, Osservatorio Astronomico di Roma, Via Frascati 33 I-00078 Monteporzio Catone, Italy
}

Panaitescu et al. 1999; Ramirez-Ruiz \& Fenimore 2000; Guetta et al.|2001; Mészáros et al.|2002; Nakar \& Piran 2002; Kino et al. 2004; Cantó et al. 2013), as well as in blazars (Sikora et al. 1994; Ghisellini 1999; Spada et al. 2001; Böttcher \& Dermer 2010; Mimica \& Alov 2010). In recent years, similar models were applied in the study of variable emission from XRBs (Kaiser et al. 2000; Miller-Jones et al. 2005; Jamil et al. 2010; Malzac 2013, 2014; Drappeau et al. 2015) as well as tidal disruption events (Wang \& Cheng 2012). See Pe'er (2014) for a review on the similarities between these objects. Indeed, the hydrodynamical properties of the shock waves as well as the colliding shells had long been investigated in the non-relativistic as well as in the relativistic regimes (Blandford \& McKee 1976; Sari \& Piran 1995).

Despite its considerable popularity, it should be stressed that it is still unclear today whether internal shocks by themselves are the leading mechanism that produces the observed signal in these objects. In the context of GRBs for example, there are two main drawbacks of this model. Firstly, the relatively low efficiency in energy conversion, as only the differential kinetic energy can be dissipated. Several authors found that the typical efficiency of energy conversion that can be expected in a multiple shell collisions is only a few - few tens of \% (Kobavashi et al. 1997; Daigne \& Mochkovitch|1998; Kumar 2000; Guetta et al. 2001; Freedman \& Waxman 2001; Ioka et al. 2006). This result, however, depends on the velocity distribution of the ejected shells, and can become substantially higher under the appropriate conditions (Beloborodov 2000; Kobavashi \& Sari 2001). The second drawback (in the context of GRBs) is accumulating evidence in recent years that a thermal component may play an important role in explaining at least part of the observed spectra in a significant minority of GRBs (Rvde 2004, 2005; Pe'er 2008; Ryde \& Pe'er 2009; Lazzati et al. 2009; Guiriec et al. 2011; Ryde et al. 2011; Axelsson et al. 2012). As this component originates from the photosphere, a dominant thermal component implies 
that a substantial energy dissipation at larger radii may be unnecessary. Nonetheless, in most cases in which a thermal component is observed, it is accompanied by an addition non-thermal part (e.g., Pe'er 2015, and references therein).

While the validity of the internal shocks model as a leading energy dissipation mechanism is uncertain, the main alternative dissipation models, namely magnetic reconnection (Coroniti 1990; Usov 1992; Thompson 1994; Drenkhahn 2002; Lyubarsky \& Kirk 2001; Drenkhahn \& Spruit 2002) suffers an even higher degree of uncertainty. For example, as the rate of reconnection depends on the magneto-hydrodynamic (MHD) turbulence, it is difficult to be assessed from first principles without detailed specification of the environment. It is therefore of no surprise that no consensus on the origin of dissipation had been achieved to date. An in depth discussion on the current observational status in GRBs and its implications appears in several recent reviews (e.g., Piran 2004; Woosley \& Bloom 2006; Mészáros 2006; Fox \& Mészáros 2006; Zhang 2007; Gehrels et al. 2009; Meszaros \& Rees 2014; Zhang 2014; Kumar \& Zhang 2015, and references therein).

Given these uncertainties, a more in depth study is needed on the underlying physics of the different models. Indeed, within the framework of the internal shocks model, one thing in common to nearly all studies carried so far is that a detailed description of the shocked plasma conditions were calculated based on the assumption that the colliding plasmas are initially cold (Sari \& Piran 1995). While the expanding plasma shells lose their energy adiabatically during the expansion, the shock waves formed in each collision substantially heat the plasma. Thus, even if initially the plasmas are cold, in a scenario of multiple collisions, in general the colliding plasmas are not expected to be cold. While this fact was considered by several authors in calculating the overall efficiency of energy conversion (Panaitescu et al. 1999; Kumar \& Piran 2000; Beloborodov 2000; Spada et al. 2001; Guetta et al. 2001; Kino et al. 2004; Jamil et al. 2010; Malzac 2014), so far no detailed description of the shocked plasma properties was calculated in the general scenario of arbitrary hot plasmas colliding at arbitrary velocities.

Such calculation is of particular importance for two reasons. First, when arbitrary hot plasmas collide, the conditions for the formation of the two shock wave system are not always fulfilled. As a result, the amount of energy dissipated in such a collision can be substantially smaller than if shock waves are formed. Second, even if shock waves are formed, the expected spectra depends on the energy density and energy per particle in the shocked region, which are in general different than in the cold plasma collision scenario (Zhang \& Mészáros 2002). Thus, in order to provide accurate calculations of the expected lightcurve and spectra, the conditions at the shocked plasma needs to be determined.

A scenario of cold shell interacting relativistically with a hot $\left(e \gg n m_{p} c^{2}\right.$, where $e$ and $n$ are the energy and number densities, $m_{p}$ is the proton mass and $c$ is the speed of light) expanding shell that was slowed down by interacting with the ambient medium was considered by Kumar \& Piran (2000). A relative Lorentz factor between the colliding shells of 1.25 was found to represent well the scenario considered in that work. The results indeed indicate that the plasma properties following the collision deviates from the plasma properties expected in the cold-cold shells collisions.

In this work, we calculate the properties of the shocked plasma following the collision of two arbitrarily hot plasma shells. We consider a simple 1-d model which enables us to provide simple analytic estimations of the thermodynamic properties of the shocked plasmas in the various regimes. As we show below, one needs to discriminate not only between the relativistic and nonrelativistic scenarios, but the analytical solutions also depend on the energy densities of the plasmas. We thus discriminate between "cold", "cool" and "hot" plasmas (see definitions in 92 below). We derive the minimum criteria for the formation of such shocks in the different scenarios, as well as the properties (velocity, energy density and energy per particle) of the shocked plasma. In $\$ 3$ we provide a full numerical solution, which can be used for arbitrary plasma properties, and further serves to validate and demonstrate the analytical approximations in the different regimes. In 94 we discuss observational consequences of the model, and in particular we show that the efficiency in energy conversion is different than previous claims, due to the need to include a pressure term. We further demonstrate how the properties of the synchrotron emission can be used to probe the properties of the colliding shells, before summarizing in $\$ 5$.

\section{BASIC SETUP}

We consider a slab of (non-magnetized) plasma shell ("slow shell"), that propagates at some arbitrary speed $\beta_{1}=v_{1} / c$ (corresponding Lorentz factor $\Gamma_{1}$ ) in the lab frame. A second plasma shell ("fast shell"), that propagates at velocity $\beta_{4}>\beta_{1}$ collides with the slow shell. At sufficiently high $\beta_{4}$, two shock waves are formed as a result of the collision: a forward shock propagating into the slow shell, and a reverse shock propagating into the fast shell. A contact discontinuity separates the shocked slow shell material from the shocked fast shell material.

Following the collision, there are four different regimes: (1) the slow shell, (2) the shocked slow shell, (3) the shocked fast shell, and (4) the fast shell material. The velocities of the four regimes are $\beta_{i},(i=1 . .4)$ and the corresponding Lorentz factors $\Gamma_{i}=\left(1-\beta_{i}\right)^{-1 / 2}$. The thermodynamical quantities: $n_{i}, e_{i}, p_{i}$ and $\omega_{i}=e_{i}+p_{i}$ (number density, internal energy density, pressure and enthalpy, respectively) are measured in each of the fluid's (shells) rest frame. We further denote the speed of the forward and reverse shock waves in the lab frame by $\beta_{f s}, \beta_{r s}$, respectively (corresponding Lorentz factors $\left.\Gamma_{f s}, \Gamma_{r s}\right)$.

The system considered therefore contains a total of 18 free parameters $\left(\beta_{i}, n_{i}, e_{i}, p_{i}[i=1 . .4], \beta_{f s}, \beta_{r s}\right)$. The shock jump conditions, namely conservations of particle, energy and momentum flux densities at each shock wave provide six equations. Two more equations are provided by equating the pressures and velocities along the contact discontinuity. Additional four equations of state, of the form $p_{i}=\left(\hat{\gamma}_{i}-1\right)\left(e_{i}-n_{i}\right)$, where $\hat{\gamma}_{i}$ is the adiabatic index in region $i$, complete a total of 12 equations connecting the velocities and thermodynamic quantities in all four regimes. Thus, by specifying a total of six boundary conditions, namely the initial velocities, num- 
ber and energy densities in the slow and fast plasma shells $\left(\beta_{1}, \beta_{4}, n_{1}, n_{4}, e_{1}, e_{4}\right)$, the velocities and thermodynamic properties of all four regions of the system are fully determined 3

In this and the following sections we provide a complete solution in the planar case. Clearly, in the most general scenario the values of all 18 parameters can only be determined numerically. However, as we show here, simple analytical solutions exist in the limiting cases of relativistic $\left(\Gamma_{4} \gg \Gamma_{1}\right)$ as well as Newtonian $\left(\beta_{4}, \beta_{1} \ll 1\right)$ plasma shells velocities. In this section we first derive the analytical solutions, before providing a few examples of the full numerical solution in $\$ 3$ below.

\subsection{Analytical Solution: Basic Equations}

For simplicity, we assume in the calculations below that the slow plasma is at rest $\left(\beta_{1}=0, \Gamma_{1}=1\right)$. This can be viewed both as a specific case, but equally as conducting the calculations in the rest frame of the slow plasma shell. Towards the end of the next section, we transform the derived results to the lab frame, in which $\Gamma_{1} \geq 1$ is arbitrary. Thus, in the calculations below, $\Gamma_{2}$ is the Lorentz factor of the shocked slow plasma (region (2)) in the rest frame of region (1), etc.

The forward shock jump conditions follow from the continuity of energy $\left(T^{01}=\omega \Gamma^{2} \beta\right)$, momentum $\left(T^{11}=\right.$ $\left.\omega \Gamma^{2} \beta^{2}+p\right)$ and particle $(n \Gamma \beta)$ flux densities in the shock frame. Here, $T^{\mu \nu}=\omega u^{\mu} u^{\nu}+p \eta^{\mu \nu}$ is the stress- energy tensor, $u^{\mu}$ is the 4 -velocity and $\eta^{\mu \nu}$ is the metric tensor. In their most general form, the forward shock jump conditions can be put in the form (Blandford \& McKee 1976; Wiersma 2007)

$$
\begin{gathered}
\frac{e_{2}}{n_{2}}=\Gamma_{2} \frac{\omega_{1}}{n_{1}}-\frac{p_{1}}{n_{2}}, \\
p_{2}-p_{1}=\frac{\left(\Gamma_{2} \beta_{2}\right)^{2} n_{2} \omega_{1}}{n_{2}-\Gamma_{2} n_{1}}
\end{gathered}
$$

Similarly, the reverse shock jump conditions are written as

$$
\begin{aligned}
\frac{e_{3}}{n_{3}} & =\bar{\Gamma}_{3} \frac{\omega_{4}}{n_{4}}-\frac{p_{4}}{n_{3}}, \\
p_{3}-p_{4} & =\frac{\left(\bar{\Gamma}_{3} \bar{\beta}_{3}\right)^{2} n_{3} \omega_{4}}{n_{3}-\bar{\Gamma}_{3} n_{4}} .
\end{aligned}
$$

Here, $\bar{\Gamma}_{3}=\Gamma_{3} \Gamma_{4}\left(1-\beta_{3} \beta_{4}\right)$ is the Lorentz factor of the shocked material in region (3) relative to the unshocked fast shell in region $(4)$, and $\bar{\beta}_{3}=\left(1-\bar{\Gamma}_{3}^{-2}\right)^{1 / 2}$ is the corresponding velocity.

\subsection{Relativistic collision}

In the ultra-relativistic case, we consider the scenario where $\Gamma_{4} \gg \Gamma_{2}=\Gamma_{3} \gg 1$. Under this assumption,

$$
\bar{\Gamma}_{3} \simeq \frac{1}{2}\left(\frac{\Gamma_{4}}{\Gamma_{3}}+\frac{\Gamma_{3}}{\Gamma_{4}}\right) \approx \frac{\Gamma_{4}}{2 \Gamma_{2}} .
$$

Since it is always true that $n_{2} \geq n_{1}$ and $n_{3} \geq n_{4}$ (and clearly $\left.\omega_{1} \geq p_{1}, \omega_{4} \geq p_{4}\right)$, it is safe to neglect the second

\footnotetext{
${ }^{3}$ We assume that the conditions are homogeneous within each regime. This approximation is reasonable as long as the initial colliding shells are not too wide. It can easily be justified in the shocked regions, which are sub-sonic.
}

terms in the right hand sides of equations 1 and 3. Using the equations of state for regions (2) and (3), with the help of the modified Equations 1 and 3, the pressures in regions (2) and (3) can be written as

$$
\begin{aligned}
& p_{2}=\left(\hat{\gamma}_{2}-1\right) n_{2}\left(\frac{e_{2}}{n_{2}}-1\right) \simeq\left(\hat{\gamma}_{2}-1\right) n_{2} \Gamma_{2} \frac{\omega_{1}}{n_{1}} ; \\
& p_{3} \simeq\left(\hat{\gamma}_{3}-1\right) \omega_{4} \bar{\Gamma}_{3} \frac{n_{3}}{n_{4}} .
\end{aligned}
$$

Using these results in Equations 2, 4, and neglecting $p_{1} \ll p_{2}$ as well as $p_{4} \ll p_{3}$ (which is correct in the ultra-relativistic limit), one obtains

$$
\frac{n_{2}}{n_{1}}=\frac{\hat{\gamma}_{2}}{\hat{\gamma}_{2}-1} \Gamma_{2} ; \quad \frac{n_{3}}{n_{4}}=\frac{\hat{\gamma}_{3}}{\hat{\gamma}_{3}-1} \bar{\Gamma}_{3} .
$$

In order to proceed, we use the fact that in the relativistic limit, the adiabatic indices are $\hat{\gamma}_{2}=\hat{\gamma}_{3}=4 / 3$. Using these results, as well as the approximation derived in Equation [5] in Equation 6, the requirement $p_{2}=p_{3}$ leads to

$$
\Gamma_{2}=\Gamma_{3} \simeq \sqrt{\frac{\Gamma_{4}}{2}}\left(\frac{\omega_{4}}{\omega_{1}}\right)^{1 / 4}, \quad \bar{\Gamma}_{3} \simeq \sqrt{\frac{\Gamma_{4}}{2}}\left(\frac{\omega_{1}}{\omega_{4}}\right)^{1 / 4} .
$$

Using Equations 1, 3 and 7, the energy per particle and the energy densities in regions (2) and (3) are given by

$$
\begin{aligned}
& \frac{e_{2}}{n_{2}} \simeq \sqrt{\frac{\Gamma_{4}}{2}} \frac{\omega_{1}^{3 / 4} \omega_{4}^{1 / 4}}{n_{1}} ; \quad \frac{e_{3}}{n_{3}} \simeq \sqrt{\frac{\Gamma_{4}}{2}} \frac{\omega_{1}^{1 / 4} \omega_{4}^{3 / 4}}{n_{4}} ; \\
& e_{2}=e_{3} \simeq \frac{\hat{\gamma}_{2}}{\hat{\gamma}_{2}-1} \frac{\Gamma_{4}}{2} \omega_{1}^{1 / 2} \omega_{4}^{1 / 2}=2 \Gamma_{4} \omega_{1}^{1 / 2} \omega_{4}^{1 / 2},
\end{aligned}
$$

where in the last line we took $\hat{\gamma}_{2}=\hat{\gamma}_{3}=4 / 3$.

The results derived in Equation 9 can further be used to set a minimum criteria on the Lorentz factor of the fast shell, $\Gamma_{4}$ that enables the existence of the two-shock system if the colliding plasma shells are initially hot. Writing $e_{i}=\omega_{i} / \hat{\gamma}_{i}+\left(\hat{\gamma}_{i}-1\right) n_{i} / \hat{\gamma}_{i} \approx \omega_{i} / \hat{\gamma}_{i}$, the requirements $e_{2} \geq e_{1}$ and $e_{3}>e_{4}$ are translated into the criteria

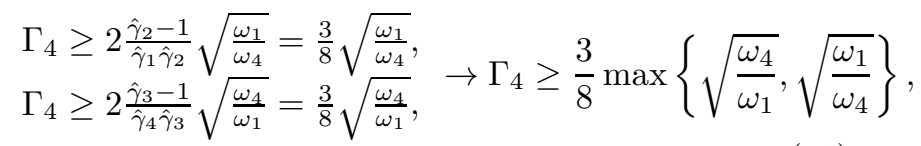

where we took $\hat{\gamma}_{i}=4 / 3$ in all four regimes, which is valid for hot plasmas. This minimum value of the Lorentz factor can be understood as follows: as the Lorentz factor of the fast shell $\left(\Gamma_{4}\right)$ decreases, eventually either the forward or reverse shock ceases to be relativistic, and the amount of energy dissipated from the (initially hot) plasma shells decreases. When this criterion is met, (at least) one of the two shock waves ceases to exist. Instead, a rarefaction wave will be created and propagate into the hot plasma, while the second shock wave could still exist.

We emphasise that the result of Equation [10] sets the minimum criteria on the Lorentz factor, originating from the physical requirement that the shock waves are capable of dissipating the shell's kinetic energy. To these criteria, one must add the underlying assumption taken in this section, that $\Gamma_{4} \gg \Gamma_{2} \gg 1$. These criteria are further validated numerically (see 93 below).

Finally, we note that a Lorentz transformation to the lab frame, in which $\Gamma_{1}^{L} \equiv \Gamma_{1} \gg 1$, yields $\Gamma_{2}^{L}=$ $\Gamma_{1} \Gamma_{2}\left(1+\beta_{1} \beta_{2}\right) \simeq 2 \Gamma_{1} \Gamma_{2}$, and similarly $\Gamma_{4}^{L} \simeq 2 \Gamma_{1} \Gamma_{4}$. The Lorentz factor of the shocked plasmas are therefore 
given by (Equation 8),

$$
\Gamma_{2}^{L}=\sqrt{\Gamma_{1}^{L} \Gamma_{4}^{L}}\left(\frac{\omega_{4}}{\omega_{1}}\right)^{1 / 4}
$$

\subsection{Newtonian collision}

We next consider the Newtonian (non-relativistic) case, in which the relative motion between the two colliding shells is non- or trans-relativistic at most. We do allow, though, the colliding plasmas to be arbitrarily hot. Similar to the relativistic treatment, we initially assume $\Gamma_{1}=1$.

In this case, it is handy to define the internal energy (excl. rest mass) $\epsilon_{1}$ by $\epsilon_{1} \equiv e_{1}-n_{1}$. When writing the energy density in region (1) as $e_{1}=n_{1}+\epsilon_{1}$, the forward shock jump conditions (equations 1, 2) can be written as a quadratic equation in the ratio of the proper densities at both sides of the forward shock wave,

$$
\begin{aligned}
& \left(\frac{n_{2}}{n_{1}}\right)^{2}\left[\Gamma_{2}\left(1+\hat{\gamma}_{1} \frac{\epsilon_{1}}{n_{1}}\right)-1\right] \\
& -\left(\frac{n_{2}}{n_{1}}\right)\left[\left(1+\hat{\gamma}_{1} \frac{\epsilon_{1}}{n_{1}}\right)\left(2+\frac{\hat{\gamma}_{2}}{\hat{\gamma}_{2}-1} \Gamma_{2}^{2} \beta_{2}^{2}\right)-\left(\Gamma_{2}+1\right)\right] \\
& +\hat{\gamma}_{1} \Gamma_{2} \frac{\epsilon_{1}}{n_{1}}=0
\end{aligned}
$$

(with a similar equation holding for the reverse shock). Note that Equation 12 is exact for all velocities. In order to obtain useful approximation in the Newtonian regime, one needs to (i) approximate $\Gamma_{2} \simeq 1+\beta_{2}^{2} / 2$; and (ii) discriminate between different regimes, based on the value of $\epsilon_{1}$ : hot, cool and cold.

In the hot regime, $\epsilon_{1} / n_{1} \gg 1$. The ratio of densities at both sides of the shock waves becomes

$$
\left(\frac{n_{2}}{n_{1}}\right)_{\text {hot }} \simeq 1+\frac{\beta_{2}}{\sqrt{\hat{\gamma}_{2}-1}}+\frac{\beta_{2}^{2}}{2\left(\hat{\gamma}_{2}-1\right)}=1+\sqrt{3} \beta_{2}+\frac{3}{2} \beta_{2}^{2}
$$

where we took $\hat{\gamma}_{1}=\hat{\gamma}_{2} \simeq 4 / 3$ in the last equality. Using this result in Equation 1 the energy density in region (2) is given by

$$
\begin{aligned}
e_{2} & \simeq \epsilon_{1}\left(1+\frac{\hat{\gamma}_{1}}{\sqrt{\hat{\gamma}_{2}-1}} \beta_{2}+\frac{\hat{\gamma}_{1} \hat{\gamma}_{2}}{2\left(\hat{\gamma}_{2}-1\right)} \beta_{2}^{2}\right) \\
& =\epsilon_{1}\left(1+\frac{4}{\sqrt{3}} \beta_{2}+\frac{8}{3} \beta_{2}^{2}\right) .
\end{aligned}
$$

Clearly, a similar equation holds for the energy density in region (3).

In the opposite, cool / cold limit, namely $\epsilon_{1} / n_{1} \ll 1$, we proceed as follows. First, in the non-relativistic limit, $\beta_{2} \ll 1$, the ratio of densities derived from the shock jump conditions (Equation 12) can be put in the form

$$
\begin{aligned}
& \left(\frac{n_{2}}{n_{1}}\right)^{2}\left[\frac{\beta_{2}^{2}}{2}+\hat{\gamma}_{1} \frac{\epsilon_{1}}{n_{1}}\left(1+\frac{\beta_{2}^{2}}{2}\right)\right] \\
& -\left(\frac{n_{2}}{n_{1}}\right)^{2}\left[\left(\frac{\hat{\gamma}_{2}+1}{\hat{\gamma}_{2}-1}\right) \frac{\beta_{2}^{2}}{2}+2 \hat{\gamma}_{1} \frac{\epsilon_{1}}{n_{1}}\left(1+\frac{\hat{\gamma}_{2}}{\hat{\gamma}_{2}-1} \frac{\beta_{2}^{2}}{2}\right)\right] \\
& +\hat{\gamma}_{1} \frac{\epsilon_{1}}{n_{1}}\left(1+\frac{\beta_{2}^{2}}{2}\right)=0 .
\end{aligned}
$$

We next discriminate between the cool case, in which $\epsilon_{1} / n_{1} \gg \beta_{2}^{2}$, and the opposite, cold case, in which $\epsilon_{1} / n_{1} \ll \beta_{2}^{2}$. In the cool scenario, the ratio of densi-
TABLE 1

Shocked Plasma VELOCITIES IN THE VARIOUS CASES.

\begin{tabular}{ll}
\hline \hline Scenario & \multicolumn{1}{c}{ Shocked shell velocities, $\beta_{2}=\beta_{3}$} \\
\hline (a) cold $\rightarrow$ cold & $\beta_{4} \frac{\sqrt{\frac{n_{4}}{n_{1}}}}{\sqrt{\frac{n_{4}}{n_{1}}}+1}$ \\
(b) cold $\rightarrow$ cool & $\beta_{4}-\left[\frac{1}{2}\left(\frac{\hat{\gamma}_{2}-1}{\hat{\gamma}_{3}-1}\right)\left(\frac{n_{1}}{n_{4}}\right)\left(\frac{\epsilon_{1}}{n_{1}}\right)\right]^{1 / 2}$ \\
(c) cold $\rightarrow$ hot & same as $(\mathrm{b})$, cold $\rightarrow$ cool \\
(d) cool $\rightarrow$ cool & $\frac{\left(\hat{\gamma}_{3}-1\right) \epsilon_{4}-\left(\hat{\gamma}_{2}-1\right) \epsilon_{1}+\left(\hat{\gamma}_{3}-1\right) \sqrt{\frac{\hat{\gamma}_{4}}{\hat{\gamma}_{4}-1}} \sqrt{n_{4} \epsilon_{4}} \beta_{4}}{\left(\hat{\gamma}_{2}-1\right) \sqrt{\frac{\hat{\gamma}_{1}}{\hat{\gamma}_{1}-1}} \sqrt{n_{1} \epsilon_{1}}+\left(\hat{\gamma}_{3}-1\right) \sqrt{\frac{\hat{\gamma}_{4}}{\hat{\gamma}_{4}-1}} \sqrt{n_{4} \epsilon_{4}}}$ \\
(e) cool $\rightarrow$ hot & $\frac{\left(\hat{\gamma}_{3}-1\right) \epsilon_{4}-\left(\hat{\gamma}_{2}-1\right) \epsilon_{1}+\left(\hat{\gamma}_{3}-1\right) \sqrt{\frac{\hat{\gamma}_{4}}{\hat{\gamma}_{4}-1}} \sqrt{n_{4} \epsilon_{4}} \beta_{4}}{\left(\hat{\gamma}_{2}-1\right) \frac{4}{\sqrt{3} \epsilon_{1}}+\left(\hat{\gamma}_{3}-1\right) \sqrt{\frac{\hat{\gamma}_{4}}{\hat{\gamma}_{4}-1} \sqrt{n_{4} \epsilon_{4}}}}$ \\
(f) hot $\rightarrow$ hot & $\frac{\epsilon_{4}-\epsilon_{1}+\frac{4}{\sqrt{3}} \epsilon_{4} \beta_{4}}{\frac{4}{\sqrt{3}}\left(\epsilon_{1}+\epsilon_{4}\right)}$
\end{tabular}

ties and the energy density in region (2) are given by

$$
\begin{aligned}
& \left(\frac{n_{2}}{n_{1}}\right)_{\mathrm{cool}} \simeq 1+\frac{1}{\sqrt{\hat{\gamma}_{1}\left(\hat{\gamma}_{2}-1\right) \frac{\epsilon_{1}}{n_{1}}}} \beta_{2}+\frac{3-\hat{\gamma}_{2}}{4 \hat{\gamma}_{1}\left(\hat{\gamma}_{2}-1\right) \frac{\epsilon_{1}}{n_{1}}} \beta_{2}^{2}, \\
& \left(e_{2}\right)_{\mathrm{cool}} \simeq n_{1}\left[1+\frac{\epsilon_{1}}{n_{1}}+\frac{1}{\sqrt{\hat{\gamma}_{2}-1}}\left(\sqrt{\hat{\gamma}_{1} \frac{\epsilon_{1}}{n_{1}}}+\frac{1}{\sqrt{\hat{\gamma}_{1} \frac{\epsilon_{1}}{n_{1}}}}\right) \beta_{2}\right] .
\end{aligned}
$$

In this case, the value of the adiabatic index is not apriori known (see discussion in section 3 below).

In the cold scenario, $\epsilon_{1} / n_{1} \ll \beta_{2}^{2}$, similar calculation yields

$$
\begin{aligned}
\left(\frac{n_{2}}{n_{1}}\right)_{\mathrm{cold}} \simeq & \left(\frac{\hat{\gamma}_{2}+1}{\hat{\gamma}_{2}-1}\right)+\frac{2 \hat{\gamma}_{1}}{\hat{\gamma}_{2}+1} \frac{\epsilon_{1}}{n_{1}}-\frac{8 \hat{\gamma}_{1}}{\left(\hat{\gamma}_{2}+1\right)\left(\hat{\gamma}_{2}-1\right)} \frac{\epsilon_{1}}{n_{1} \beta_{2}^{2}} \\
= & 4+\left(\frac{5}{4}-\frac{15}{2 \beta_{2}^{2}}\right)\left(\frac{\epsilon_{1}}{n_{1}}\right), \\
\left(e_{2}\right)_{\mathrm{cold}} \simeq & n_{1}\left[\left(\frac{\hat{\gamma}_{2}+1}{\hat{\gamma}_{2}-1}\right)\left(1+\frac{\beta_{2}^{2}}{2}\right)-\frac{8 \hat{\gamma}_{1}}{\left(\hat{\gamma}_{2}+1\right)\left(\hat{\gamma}_{2}-1\right)}\left(\frac{\epsilon_{1}}{n_{1} \beta_{2}^{2}}\right)\right. \\
& \left.+\frac{3 \hat{\gamma}_{1}+1}{\hat{\gamma}_{2}+1}\left(\frac{\epsilon_{1}}{n_{1}}\right)\right] \\
= & n_{1}\left[4\left(1+\frac{\beta_{2}^{2}}{2}\right)+\left(\frac{9}{4}-\frac{15}{2 \beta_{2}^{2}}\right)\left(\frac{\epsilon_{1}}{n_{1}}\right)\right],
\end{aligned}
$$

where we used $\hat{\gamma}_{1}=\hat{\gamma}_{2}=5 / 3$ in the last equality. We further point out that given an arbitrary value of $0<$ $\left(\epsilon_{1} / n_{1}\right)<1$, for very small relative velocities between the plasma shells the plasma can be considered as "cool", while at higher velocities it can be regarded as "cold".

The results presented in Equations 12-17]are of course symmetric with respect to the reverse shock, and are obtained by replacing quantities in regions (1), (2) with those in regions (4), (3), respectively, and exchanging $\beta_{2}$ with $\bar{\beta}_{3} \simeq \beta_{4}-\beta_{2}$. Using these replacements, one can use the requirement $p_{2}=p_{3}$ to determine the shocked plasma velocity, $\beta_{2}=\beta_{3}$ as a function of the colliding plasmas parameters $\left(n_{1}, e_{1}, n_{4}\right.$ and $\left.e_{4}\right)$ as well as their relative velocities $\beta_{4}$ in the different regimes. The results of the various scenarios are summarized in Table 1.

The results in Table 1 can also be used to put constraints on the minimum relative velocities between the shells $\left(\beta_{4}\right)$ that enables the formation of the double shock structure. These limits originate from the requirements (a) $\beta_{2} \geq 0$ and (b) $\bar{\beta}_{3}=\beta_{4}-\beta_{2} \geq 0$. In the first scenario considered in Table 1 that of interaction between two cold plasmas, $\beta_{2}$ is always smaller than $\beta_{4}$ and therefore 
there is no restriction: a double shock structure will always form, for each value of $\beta_{4}>0$. However, this is the exceptional case: in all other scenarios, in which at least one of the shells is not completely cold, such a restriction does exist. If $\beta_{4}$ is smaller then the minimum value set by $n_{1}, n_{4}, \epsilon_{1}$ and $\epsilon_{4}$, the ram pressure cannot compensate for the excess energy gained by thermalization at the shock front. In these cases, two shocks cannot form. Rather, similar to the relativistic case, a rarefaction wave will form, which will gradually modify the properties of one of the shells.

\section{NUMERICAL SOLUTION}

In order to validate the analytical approximations presented in Section 2 above as well as to investigate the intermediate velocity (trans-relativistic) regime, we wrote a numerical code which solves the dynamical conditions at each of the four regimes - unshocked and shocked plasma shells that follow the collision of two plasma shells. The code simultaneously solves the set of twelve coupled equations: three shock jump conditions each for the forward and reverse shock waves, equating the pressure and velocity along the contact discontinuity and four equations of state. The results are obtained for a given set of six initial conditions: velocity, number and energy densities in regions (1) and (4), the unshocked plasmas.

\subsection{Determination of the adiabatic indices in the different regimes}

In order to account for the energy dependence of the adiabatic indices $\hat{\gamma}_{i}$ in each of the four regimes, we use the prescription derived by Service (1986), which is accurate to $10^{-5}$. Since the classical gas law, $p_{i}=n_{i} T_{i}$ holds exactly in all regimes, one can write

$$
\frac{e_{i}}{n_{i}}=T_{i}\left(\frac{e_{i}+p_{i}}{p_{i}}-1\right) .
$$

We use the approximation derived by Service (1986),

$$
\begin{aligned}
\frac{p_{i}}{e_{i}+p_{i}}= & 0.36 y+0.036346 y^{2}-0.088763 y^{3} \\
& -0.047698 y^{4}-0.083547 y^{5}+0.073662 y^{6},
\end{aligned}
$$

where

$$
y \equiv \frac{T_{i}}{0.36+T_{i}} .
$$

The results of Equation 19 are tabulated. Thus, for a given ratio $e_{i} / n_{i}$, we use the tabulated results in Equation 18 to infer the temperature $T_{i}$ in region $i$.

Once the temperature is known, at a second step the adiabatic index in each regime is calculated using

$$
\begin{aligned}
\hat{\gamma}_{i}=\frac{1}{3} & \left(5-1.21937 z+0.18203 z^{2}-0.96583 z^{3}\right. \\
& \left.+2.32513 z^{4}-2.39332 z^{5}+1.07136 z^{6}\right)
\end{aligned}
$$

where

$$
z \equiv \frac{T_{i}}{0.24+T_{i}}
$$

Calculation of the dynamical properties of the plasmas in the different regimes is done as follows. We first guess a value of the shocked plasma velocity (more precisely, of $\Gamma_{2} \beta_{2}=\Gamma_{3} \beta_{3}$ ), and solve for the two shock jump conditions. The value of $\Gamma_{2} \beta_{2}$ is then varied, until the pressures at each side of the contact discontinuity are equal.

In order to determine the adiabatic index in the shocked regions, for each value of $\Gamma_{2} \beta_{2}$ the shock jump conditions are solved in iterative way. Following an initial guess of $\hat{\gamma}_{2}, \hat{\gamma}_{3}$, the shock jump conditions are solved and the values of the specific energies $e_{2} / n_{2}$ and $e_{3} / n_{3}$ are determined. The values of the adiabatic index are then re-calculated, and the calculation is repeated with the new value. We found that convergence is typically very quick, within few iterations at most.

\subsection{Numerical results}

Examples of the numerical results, together with the analytical approximations in the different regimes are presented in Figures 1 - 3 .

In Figure 1, we calculate the dynamical and thermal properties in all four regimes following the collision of two cold plasma shells. The first (slow) shell is characterized by density $n=1 \mathrm{~cm}^{-3}$ and zero internal energy $\left(e_{1}=\right.$ $\left.n_{1} m_{e} c^{2}\right)$. The fast plasma shell is characterized by higher density of $n_{4}=100 \mathrm{~cm}^{-3}$ and is similarly cold, $e_{4}=$ $n_{4} m_{e} c^{2}$. The density contrast is chosen to be 100 for presentation purposes. We further chose the slow plasma to be motionless, $\beta_{1}=0$. The results are presented as a function of $\Gamma_{4} \beta_{4}$, where $\beta_{4}$ is the relative velocity between the shells.

For cold plasmas as considered in Figure 1, there is no lower limit on $\beta_{4}$, i.e., the two shock system always forms, for any value of $\beta_{4}>0$. This system of cold plasmas is in fact identical to the one considered already by Sari \& Piran (1995). In the upper left panel of Figure 1 we show the shocked plasma velocity, $\left(\Gamma_{2} \beta_{2}\right)$, in the rest frame of the slow shell as well as the same velocity in the rest frame of the unshocked, fast plasma in region (4), denoted by $\left(\bar{\Gamma}_{3} \bar{\beta}_{3}\right)$. The asymptotic approximations in the relativistic (Equations 8) and non-relativistic (Table 1 (a)) regimes are given by the dashed and dash-dotted lines. In producing the non-relativistic approximation of the velocities, we replace $\beta_{4}$ with $\Gamma_{4} \beta_{4}$. The results show excellent agreement - better than $\sim 10 \%$ for $\Gamma_{4} \beta_{4} \leq 2$.

In upper right and lower left panels of Figure 1 we show the energy densities and the energy per particle $\left(e_{i} / n_{i}\right)$ in the shocked plasma regions (2) and (3) as a function of $\Gamma_{4} \beta_{4}$. The analytic approximations in the relativistic regime (Equation 9) and non-relativistic regime (Equation 17) again provide an excellent description of the thermodynamical properties of the plasma. The transition between the non-relativistic and relativistic regimes occurs for those values of $\Gamma_{4} \beta_{4}$ in which $\Gamma_{2} \beta_{2} / \bar{\Gamma}_{3} \bar{\beta}_{3}$ becomes relativistic. Finally, in the lower right panel of Figure 11 we show the adiabatic indices in the different regimes. While clearly $\hat{\gamma}_{1}=\hat{\gamma}_{4}=5 / 3$, the adiabatic indices of the shocked plasma are gradually changing as $\Gamma_{4} \beta_{4}$ increases, and the shocked plasma heats.

In Figure 2 we consider a more complicated scenario, that of a collision between two cool shells. We chose as parameters $n_{1}=99.99 \mathrm{~cm}^{-3}, \epsilon_{1}=0.01 \mathrm{erg} \mathrm{cm}^{-3}$ (namely, $e_{1}=100 \mathrm{erg} \mathrm{cm}-3$ ), $n_{4}=4.99 \mathrm{~cm}^{-3}$ and $\epsilon_{4}=$ $0.01 \mathrm{erg} \mathrm{cm}^{-3}$. Similar to the previous example, we took $\beta_{1}=0$, namely slow shell at rest. These values are chosen for presentation purposes, as we want to ensure a good contrast of the shocked plasma properties between the 

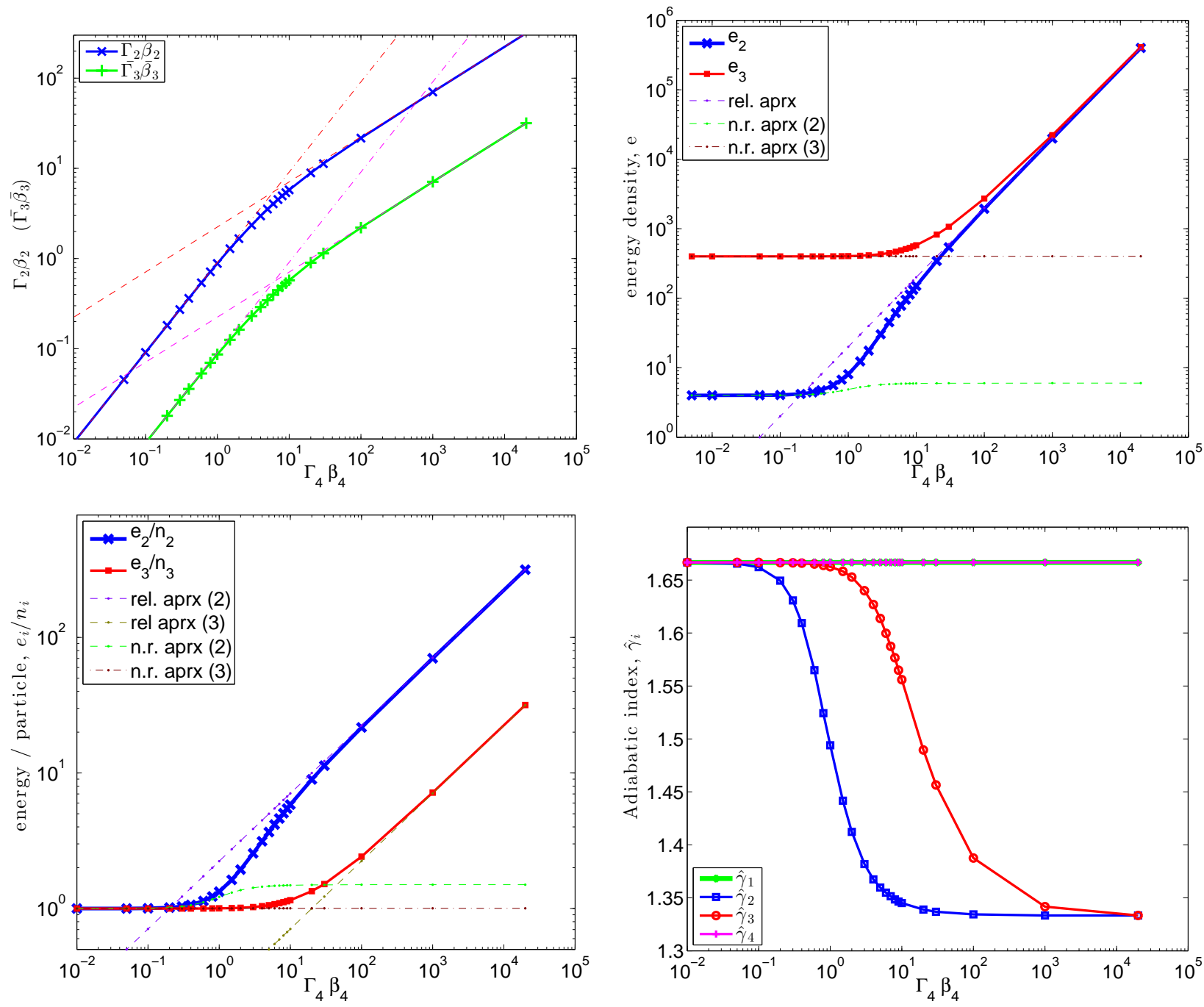

FIG. 1. - Velocities and thermodynamic properties of the shocked plasma following collision between two cold plasma shells $\left(\epsilon_{i}=0\right.$, $i=1,4)$. Parameters considered are $n_{1}=1 \mathrm{~cm}^{-3}, \epsilon_{1}=0, n_{4}=100 \mathrm{~cm}^{-3}, \epsilon_{4}=0, \beta_{1}=0$. Plasma parameters are shown as a function of the fast shell initial velocity, $\Gamma_{4} \beta_{4}$. Upper left: Velocities of the shocked plasma regions (2) and (3), as measured in the rest frame of slow plasma shell in region (1) $\left(\Gamma_{2} \beta_{2}\right)$ and the rest frame of the fast plasma shell in region $(4),\left(\bar{\Gamma}_{3} \bar{\beta}_{3}\right)$. Upper right: Energy densities in the shocked plasma regions (2) and (3). Lower left: Energy per particle, $e_{i} / n_{i}$ in the shocked plasma regions (2) and (3). Lower right: Adiabatic indeices $\hat{\gamma}_{i}$ in the four different regimes.

different regimes.

The velocities of the shocked plasma regions (2) and (3) as measured in the rest frames of the slow plasma shell $\left(\Gamma_{2} \beta_{2}\right)$ and the fast plasma shell $\left(\bar{\Gamma}_{3} \bar{\beta}_{3}\right)$ are shown in the upper left panel of Figure 2. The analytical approximation in the relativistic regime (Equation 8) and the non-relativistic regime (Table $1(\mathrm{~d})$ ) provide excellent aproximations in the two regimes. The decay of the analytical approximation to $\bar{\beta}_{3}=\beta_{4}-\beta_{2}$ around $\Gamma_{4} \sim 1$ arises from the use of $\Gamma_{4} \beta_{4}$ in the calculation of $\beta_{2}$.

The ratio of densities across the forward shock is shown in the upper right panel of Figure 2, together with the analytic approximations. There are clearly three distinct regimes. First, there is the relativistic regime, $\Gamma_{2} \gg 1$. In this regime, the density ratio is well approximated by the results given in Equation 7 A second regime is the non-relativistic, "cold" regime, namely $\beta_{2} \geq \sqrt{2 \epsilon_{1} / n_{1}}=0.014$ (in the considered scenario), in which the density ratio is well approximated by Equation
17. Finally, when $\beta_{2} \ll \sqrt{2 \epsilon_{1} / n_{1}}$, the approximation in the "cool" regime given in Equation 16 provides a good fit to the density ratio. These same three regimes are also clearly observed when considering the energy densities of the shocked plasma in Figure2 (lower left). Interestingly, when considering the ratio $e_{i} / n_{i}$ (Figure2, lower right) in the non-relativistic regime, the cool and cold approximations can be combined to provide a good approximation which reads $e_{2} / n_{2} \simeq 1+\left(\epsilon_{1} / n_{1}\right)+\left(\Gamma_{2} \beta_{2}\right)^{2} / 2$. In the relativistic regime, this ratio is well described by Equation 9.

In Figure 3 we provide a third example, that of a collision between two initially hot plasma shells. As initial parameters, we chose $n_{1}=1 \mathrm{~cm}^{-3}, e_{1}=15 \mathrm{erg} \mathrm{cm}^{-3}$,

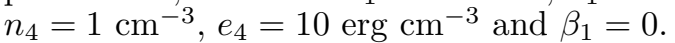

For this choice of parameters, the results of Table 1 (f) show a minimum value of $\beta_{4}$, below which two shock waves cannot form: For $\beta_{4}=\sqrt{3}\left(\epsilon_{1}-\epsilon_{4}\right) / 4 \epsilon_{4} \simeq 0.24$, $\beta_{2} \rightarrow 0$. This is clearly demonstrated in Figure 3 [top left]. At larger relative velocity, the results in Equa- 

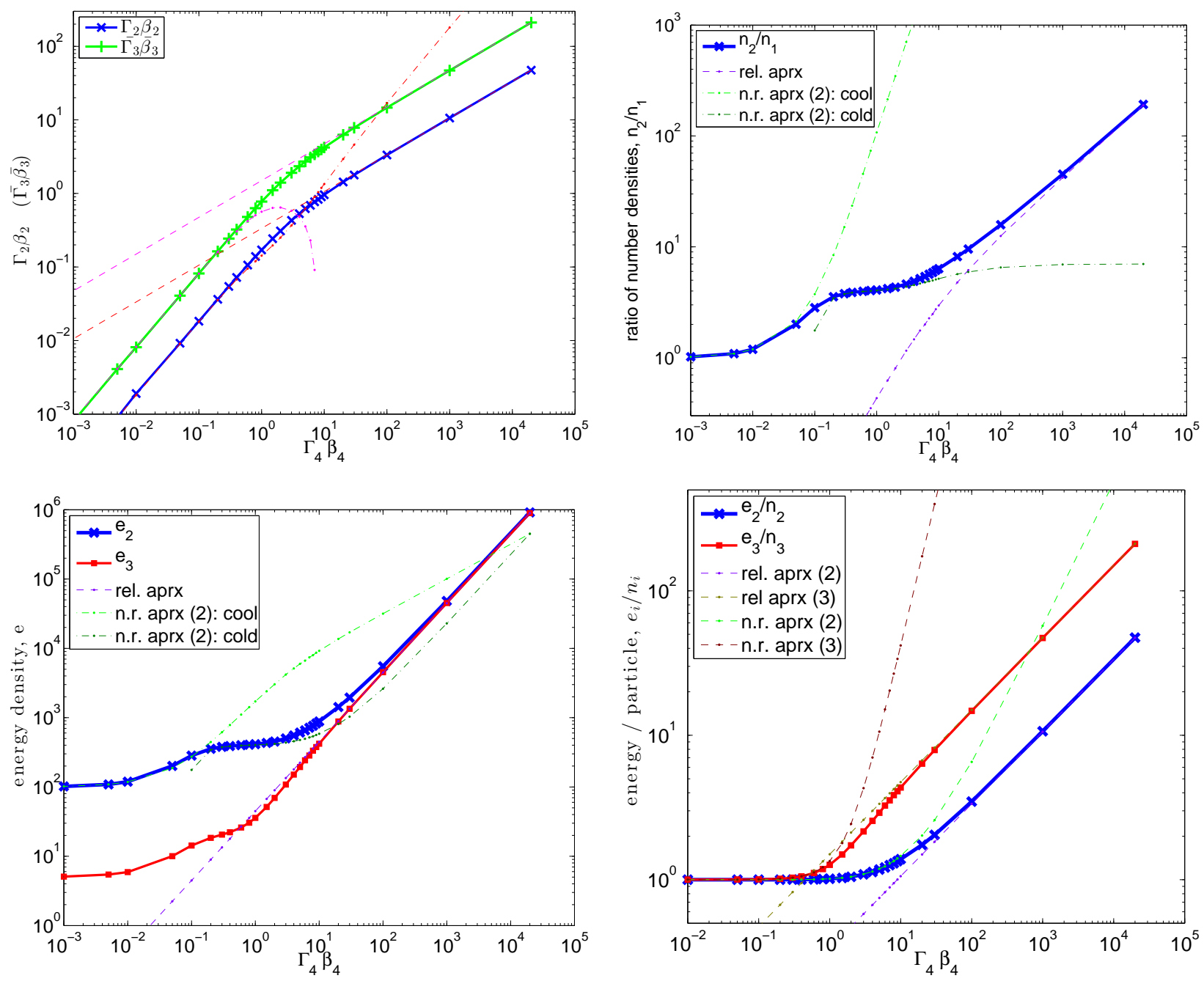

FIG. 2.- Velocities and thermodynamic properties of the shocked plasma following collision between two cool plasma shells $\left(\epsilon_{i} / n_{i} \ll 1\right.$, $i=1,4)$. Parameters considered are $n_{1}=99.99 \mathrm{~cm}^{-3}, \epsilon_{1}=0.01 \mathrm{erg} \mathrm{cm}{ }^{-3}, n_{4}=4.99 \mathrm{~cm}^{-3}, \epsilon_{4}=0.01 \mathrm{erg} \mathrm{cm} \mathrm{cm}^{-3}, \beta_{1}=0$. Plasma parameters are shown as a function of the fast shell initial velocity, $\Gamma_{4} \beta_{4}$. Upper left: velocities of the shocked plasma (same as in Figure 1 [upper left]), for collision of cool plasma shells. Upper right: Ratio of number densities across the forward shock, $n_{2} / n_{1}$. Lower left: Energy densities in the shocked plasma regions (2) and (3). Lower right: Energy per particle, $e_{i} / n_{i}$ in the shocked plasma regions (2) and (3).

tion 8 and Table 1 (f) provide an excellent approximation to the shocked plasma velocity. The ratio of number densities across the forward shock, $n_{2} / n_{1}$ (Figure 3. top right) is well approximated by the analytical approximations in Equation 7 (relativistic) and 13 (nonrelativistic). Similarly, the energy per particle in the shocked regions (2) and (3) shown in the bottom left panel of Figure 3 are well approximated by the analytical result in Equation 9 in the relativistic regime, and by $e_{2} / n_{2} \simeq\left(e_{1} / n_{1}\right)\left(1+\Gamma_{2} \beta_{2} / \sqrt{3}+\left(\Gamma_{2} \beta_{2}\right)^{2} / 6\right)$ in the nonrelativistic regime, which is readily derived from Equations 13 and 14

\section{OBSERVATIONAL CONSEQUENCES}

\subsection{Efficiency in kinetic energy dissipation}

The calculations above enable us to determine the efficiency of kinetic energy conversion during two shell collisions. Various authors have calculated this effciency using an integral approach, namely by determining the merged shell bulk Lorentz factor assuming a plastic collision between the two shells, and using conservations of energy and momentum. For example, Kobavashi et al. (1997) and Malzad (2014) considered cold plasma shells, while Spada et al. (2001) and Jamil et al. (2010) generalized the result to hot plasmas.

Using the formulation developed here, we can generalize these results. The calculation is done in the rest frame of the shocked plasma. In this frame, the slow shell in region (1) is seen to have a Lorentz factor $\tilde{\Gamma}_{1}$ (corresponding velocity $\left.\tilde{\beta}_{1}\right)$, where $\tilde{\Gamma}_{1} \tilde{\beta}_{1}=\Gamma_{1} \Gamma_{2}\left(\beta_{1}-\beta_{2}\right)$. The transfer of momentum from the slow shell (region (1)) to the shocked plasma (region (2)), assuming a planar symmetry along the $x$ direction is given by

$$
d P^{x}(1)=\int d V_{1}^{\prime} T^{01}=\int d V_{1}^{\prime} \omega_{1} \tilde{\Gamma}_{1}^{2} \tilde{\beta}_{1}=\int d V_{1} \omega_{1} \tilde{\Gamma}_{1} \tilde{\beta}_{1} .
$$

Here, $d V_{1}^{\prime}=d V_{1} / \tilde{\Gamma}_{1}$ is the volume element of material in region (1) that crosses the forward shock into region (2) per unit time, as seen in the rest frame of shocked region (2), and $d V_{1}$ is the same volume element as measured in the rest frame of region (1). 

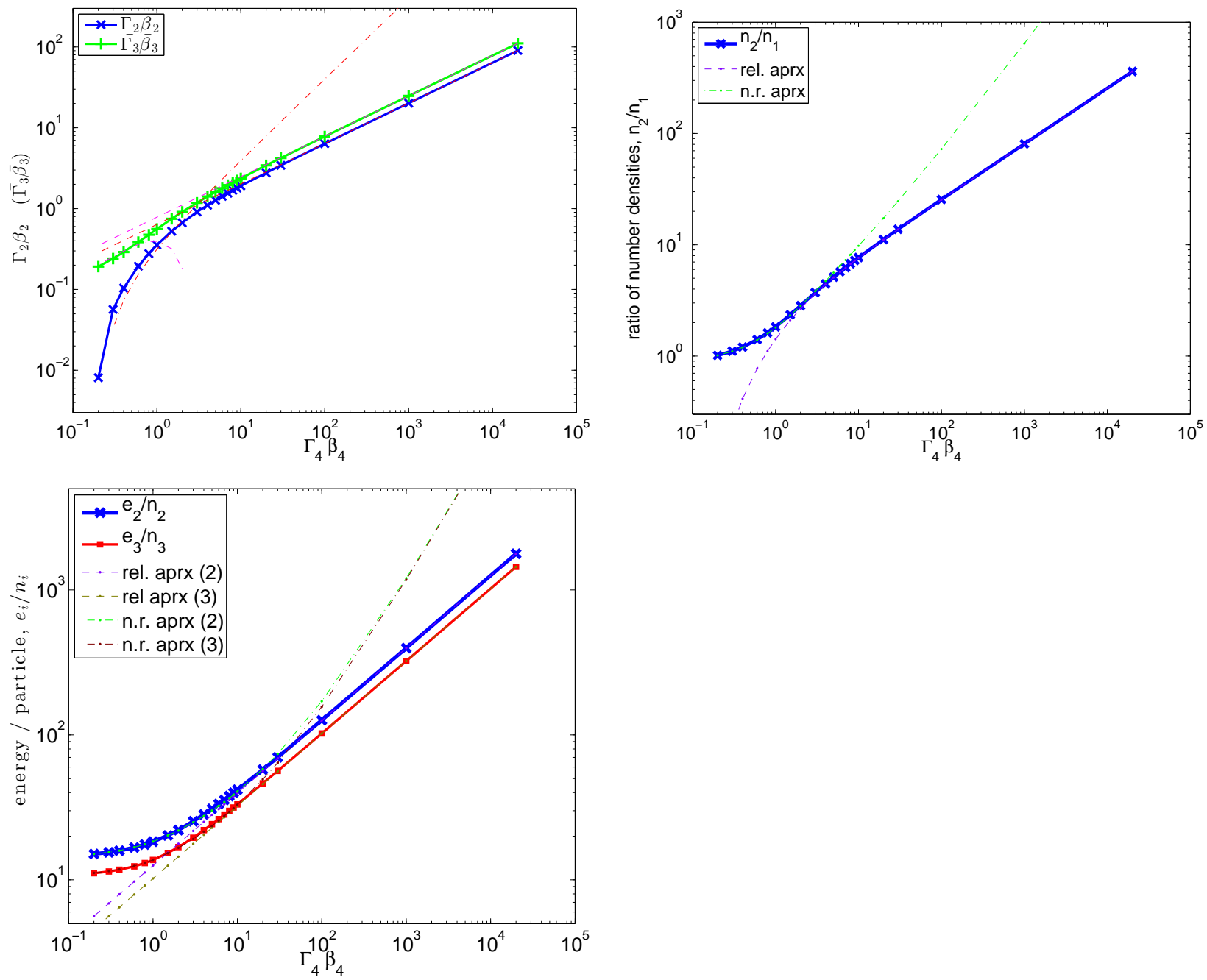

FIG. 3.- Velocities and thermodynamic properties of the shocked plasma following collision between two initially hot $\left(\epsilon_{i} / n_{i} \gg 1\right)$ plasma shells. Parameters considered are $n_{1}=1 \mathrm{~cm}^{-3}, e_{1}=15 \mathrm{erg} \mathrm{cm}{ }^{-3}, n_{4}=1 \mathrm{~cm}^{-3}, e_{4}=10 \mathrm{erg} \mathrm{cm}{ }^{-3}, \beta_{1}=0$. Plasma parameters are shown as a function of the fast shell initial velocity, $\Gamma_{4} \beta_{4}$. Upper left: Velocities of the shocked plasma (same as in Figure[1] [upper left]), for collision of two hot plasma shells. Upper right: Ratio of number densities across the forward shock, $n_{2} / n_{1}$. Lower left: Energy per particle, $e_{i} / n_{i}$ in the shocked plasma regions (2) and (3).

Using $\omega_{1}=n_{1}+\hat{\gamma}_{1} \epsilon_{1}$, as well as $d M_{1}=\int d V_{1} n_{1}$ and $d E_{t h, 1}=\int d V_{1} \epsilon_{1}$, the momentum transfer rate can be written as

$$
d P^{x}(1)=\left(d M_{1}+\hat{\gamma}_{1} d E_{t h, 1}\right) \tilde{\Gamma}_{1} \tilde{\beta}_{1},
$$

where we assumed that the velocities are not changed during the shock propagation.

A similar calculation holds for the momentum transfer from the fast plasma (region (4)), which could be written as

$$
d P^{x}(4)=\left(d M_{4}+\hat{\gamma}_{4} d E_{t h, 4}\right) \bar{\Gamma}_{3} \bar{\beta}_{3},
$$

where $\bar{\Gamma}_{3} \bar{\beta}_{3}=\Gamma_{4} \Gamma_{2}\left(\beta_{4}-\beta_{2}\right)$. Equating the momentum transfer in both sides leads to the velocity at the center of mass frame, which is the shocked fluid frame as long as both shockes exist,

$$
\beta_{2}=\frac{\Gamma_{1} \beta_{1}\left(d M_{1}+\hat{\gamma}_{1} d E_{t h, 1}\right)+\Gamma_{4} \beta_{4}\left(d M_{4}+\hat{\gamma}_{4} d E_{t h, 4}\right)}{\Gamma_{1}\left(d M_{1}+\hat{\gamma}_{1} d E_{t h, 1}\right)+\Gamma_{4}\left(d M_{4}+\hat{\gamma}_{4} d E_{t h, 4}\right)} .
$$

In the ultra-relativistic case, $\Gamma_{4} \gg \Gamma_{2} \gg 1$ this can be written as

$$
\Gamma_{2} \simeq\left(\frac{\Gamma_{1}\left(d M_{1}+\hat{\gamma}_{1} d E_{t h, 1}\right)+\Gamma_{4}\left(d M_{4}+\hat{\gamma}_{4} d E_{t h, 4}\right)}{\left(d M_{1}+\hat{\gamma}_{1} d E_{t h, 1}\right) / \Gamma_{1}+\left(d M_{4}+\hat{\gamma}_{4} d E_{t h, 4}\right) / \Gamma_{4}}\right)^{1 / 2} .
$$

This result differs from the result that appears in Spada et al. (2001) (their Equation (4)) as well as in Jamil et al. (2010), by the inclusion of the adiabatic indices $\hat{\gamma}_{i}$ that multiply the thermal energies, which are omitted in these works. These can be traced back to the inclusion of the pressure term in the shocked plasma.

We further point out that equating the momenta transfer from regions (1) and (4) using equations 24 and 25 in the relativistic case, would retrieve back Equation 8. These results imply that the efficiency of kinetic energy conversion as calculated in Spada et al. (2001) and Jamil et al. (2010) hold, provided that the final Lorentz factor is calculated using Equation 27.

\subsection{Basic scalings of synchrotron emission}

The heated shocked plasma will radiate its energy. The observed signal can therefore be used as a probe of the 
initial, unshocked plasma shells properties. Full radiative calculations require additional parameters, such as the exact value of the magnetic field as well as assumptions about the radiating paricles distribution in the shocked plasma regions, and are therefore left for a future work.

Here, we provide some basic scaling laws of the characteristic frequencies expected from synchrotron emission, which is likely the easiest (and most commonly discussed) signal that can be detected, and can therefore be used to probe the plasma conditions. These are particularly simple in the relativistic regime, where the plasma is substantially heated by the shock waves. We therefore focus here in the relativistic regime.

We scale the properties of the synchrotron emission in region $i$ by adopting the common assumption that magnetic fields are generated by the shock waves, and that the generated magnetic energy density is some constant fraction of the post-shock thermal energy density, namely $B_{i}^{2} \propto e_{i}$. Furthermore, we assume that the electrons carry some constant fraction of the proton energy, resulting in electron's Lorentz factor $\gamma_{e l, i} \propto\left(e_{i} / n_{i}\right)$. As the characteristic synchrotron emission frequency is $\nu_{s y n, i} \propto B_{i} \gamma_{e l, i}^{2}$, one finds the scaling

$$
\frac{\nu_{s y n, 2}}{\nu_{s y n, 3}} \propto \frac{e_{2}^{1 / 2}\left(\frac{e_{2}}{n_{2}}\right)^{2}}{e_{3}^{1 / 2}\left(\frac{e_{3}}{n_{3}}\right)^{2}}=\left(\frac{n_{4}}{n_{1}}\right)^{2}\left(\frac{\omega_{1}}{\omega_{4}}\right) .
$$

If we denote by $\Delta_{1}$ and $\Delta_{4}$ the (comoving) widths of the colliding shells, the total number of radiating electrons is $N_{1} \propto n_{1} \Delta_{1}$ and $N_{4} \propto n_{4} \Delta_{4}$ (under the 1-d assumption). Since the total observed power is $P_{\text {syn }} \propto N B^{2} \gamma_{\text {el }}^{2}$ (Rybicki \& Lightman 1979), the ratio of synchrotron power between the two shocked regions is therefore

$$
\frac{P_{\text {syn }, 2}}{P_{\text {syn }, 3}}=\frac{n_{1} \Delta_{1} e_{2}\left(\frac{e_{2}}{n_{2}}\right)^{2}}{n_{4} \Delta_{4} e_{3}\left(\frac{e_{3}}{n_{3}}\right)^{2}}=\left(\frac{\Delta_{1}}{\Delta_{4}}\right)\left(\frac{n_{4}}{n_{1}}\right)\left(\frac{\omega_{1}}{\omega_{4}}\right) .
$$

In the relativistic scenario, the observed time scale for the forward shock wave to cross the slow plasma shell is $\sim \Delta_{1} \Gamma_{1} / c$, while the time scale of the reverse shock to cross the fast plasma is $\approx \Delta_{4} \Gamma_{2}^{2} / \Gamma_{4} c \sim 2 \Gamma_{1}\left(\omega_{4} / \omega_{1}\right) \Delta_{4} c$ (e.g., Sari \& Piran 1995). Thus, the observed ratio of the time scale of existence of the two shock waves is

$$
\frac{t_{f s}}{t_{r s}}=\left(\frac{\Delta_{1}}{\Delta_{4}}\right)\left(\frac{\omega_{1}}{\omega_{4}}\right) .
$$

These results imply that identification of the ratios of the three main characteristics of synchrotron emission from the forward and reverse shock waves, namely the characteristic frequency, total power and time scales, are sufficient to provide direct information about the ratio of number densities, enthalpies and initial sizes of the colliding shells. Interestingly, in the ultra-relativistic limit, these results are independent on the unknown Lorentz factor. As we showed above, using these initial conditions one can calculate the properties of the merged shell. Therefore, direct observations of multiple shell collisions could provide information about two key ingredients. The first is the initial conditions of the ejected shells, hence the properties of the inner engine. The second is the temporal, hence spatial evolution (adiabatic losses) of the merged shell.

\section{SUMMARY AND DISCUSSION}

In this work, we considered the collision of two plasma shells, as is expected in the "internal shock" model. We generalized previous treatments of the problem by considering plasmas which can be arbitrarily hot. This is a natural consequence of the internal shocks scenario, as, after the first collision, the merged shell is inevitably hot (and can be very hot if the shells are relativistic, see Equation 91). We point out that while in between collisions the colliding shells lose their energy adiabatically, the decrease in temperature (or energy per particle) is $(e / n) \propto T \propto r^{-2 / 3}$, and thus even if the internal collisions occur within a range of several orders of magnitude in radii, adiabatic cooling is not sufficient to completely cool the plasma shells.

We derived analytical approximations for the shocked shell velocities in both the relativistic (Equation 8) and non-relativistic (Table 1) regimes. A very important result we found is that in the general scenario (as opposed to the cold scenario) there is a minimum relative velocity, or Lorentz factor, that enables the formation of the two shock system (Equation 10). The physical reason for this is the requirement of the ram pressure to exceed the pressure associated with the excess of thermal energy caused by the shock. If this criterion is not met, only a single shock wave is expected, while a rarefaction wave will propagate into the hotter plasma. In this case, we expect the radiative signal to be much weaker.

We further provided analytical expressions for the energy density and for the energy per particle in the shocked region. We found that for non-relativistic collision, one needs to discriminate between three scenarios: "hot" plasma, for which $\epsilon / n \gg 1$, "cool" plasmas for which $1 \gg \epsilon / n \gg \beta^{2}$, and "cold" plasma, for which $1 \gg \beta^{2} \gg \epsilon / n$. We provided the analytical expressions for thermodynamical properties of the shocked plasma in each of these cases.

We discussed several observational consequences of the dynamical results. We showed that in calculating the final Lorentz factor of the merged shell, hence the efficiency of kinetic energy dissipation, one needs to consider the pressure of the shocked plasma. We provided the basic scaling laws of synchrotron emission in the ultrarelativistic regime, and showed that measurements of the peak energy, flux and time scale of emission enables one to deduce important information about the initial shells properties, as well as the spatial evolution of the propagating shells.

The results provided here emphasise the fact that the properties of the shocked plasma depend not only on the relative velocities between the colliding plasma shells, but also on the energy per particle in each colliding shell. These results are therefore important in the study of signals from multiple collisions that are expected in various environments, such as GRBs, XRBs and AGNs. Furthermore, our numerical results are particularly useful for probing the plasma properties in the trans-relativistic regime, which is likely the dominant regime in XRBs and possibly AGNs. As we demonstrated in $\$ 3$ above, while 
no simple analytical expressions exist in this regime, still reasonable analytical fits do exist, and can be very useful in understanding the underlying properties of these objects.

The results obtained in this work imply that the overall efficiency of kinetic energy dissipation in a multiple shock scenario is in general different than previous calculations that considered collisions between cold shells (Kobavashi et al. 1997; Daigne \& Mochkovitch 1998; Lazzati et al. 1999; Kumar 2000; Beloborodov 2000; Guetta et al. 2001; Ioka et al. 2006). In a realistic scenario of hot shells, when estimating the efficiency in multiple shells collisions, one needs to consider: (i) the properties of each shell immediately after the colli- sion; (ii) the adiabatic cooling of the shells in between the collisions; and (iii) adiabatic expansion of each shell in between collisions, which results in differential velocity field within the expanding shell Kobavashi \& Sari 2001). In this work we focus on part (i) of this problem. We leave a complete treatment of a multiple shell collision scenario for a future work.

AP wishes to thank Damien Bégué, Felix Ryde, Ralph Wijers and Bing Zhang for useful comments. This research was partially supported by the European Union Seventh Framework Programme (FP7/2007-2013) under grants agreement $\mathrm{n}^{\circ} 618499$ (AP) and $\mathrm{n}^{\circ} 322259$ (PC).

\section{REFERENCES}

Axelsson, M., Baldini, L., Barbiellini, G., et. al. 2012, ApJ, 757, L31

Beloborodov, A. M. 2000, ApJ, 539, L25

Blandford, R. D. \& McKee, C. F. 1976, Physics of Fluids, 19, 1130

Böttcher, M. \& Dermer, C. D. 2010, ApJ, 711, 445

Cantó, J., Lizano, S., Fernández-López, M., González, R. F., \& Hernández-Gómez, A. 2013, MNRAS, 430, 2703

Coroniti, F. V. 1990, ApJ, 349, 538

Daigne, F. \& Mochkovitch, R. 1998, MNRAS, 296, 275

Drappeau, S., Malzac, J., Belmont, R., Gandhi, P., \& Corbel, S. 2015, MNRAS, 447, 3832

Drenkhahn, G. 2002, A\&A, 387, 714

Drenkhahn, G. \& Spruit, H. C. 2002, A\&A, 391, 1141

Fender, R. P. 2001, MNRAS, 322, 31

Fenimore, E. E., Madras, C. D., \& Nayakshin, S. 1996, ApJ, 473, 998

Fox, D. B. \& Mészáros, P. 2006, New Journal of Physics, 8, 199

Freedman, D. L. \& Waxman, E. 2001, ApJ, 547, 922

Gehrels, N., Ramirez-Ruiz, E., \& Fox, D. B. 2009, ARA\&A, 47, 567

Ghisellini, G. 1999, Astronomische Nachrichten, 320, 232

Guetta, D., Spada, M., \& Waxman, E. 2001, ApJ, 557, 399

Guiriec, S., Connaughton, V., Briggs, M. S., et. al. 2011, ApJ, 727, L33

Ioka, K., Toma, K., Yamazaki, R., \& Nakamura, T. 2006, A\&A, 458,7

Jamil, O., Fender, R. P., \& Kaiser, C. R. 2010, MNRAS, 401, 394

Kaiser, C. R., Sunyaev, R., \& Spruit, H. C. 2000, A\&A, 356, 975

Kino, M., Mizuta, A., \& Yamada, S. 2004, ApJ, 611, 1021

Kobayashi, S., Piran, T., \& Sari, R. 1997, ApJ, 490, 92

Kobayashi, S. \& Sari, R. 2001, ApJ, 551, 934

Kumar, P. 2000, ApJ, 538, L125

Kumar, P. \& Piran, T. 2000, ApJ, 532, 286

Kumar, P. \& Zhang, B. 2015, Phys. Rep., 561, 1

Lazzati, D., Ghisellini, G., \& Celotti, A. 1999, MNRAS, 309, L13

Lazzati, D., Morsony, B. J., \& Begelman, M. C. 2009, ApJ, 700, L47

Lyubarsky, Y. \& Kirk, J. G. 2001, ApJ, 547, 437

Malzac, J. 2013, MNRAS, 429, L20

-. 2014, MNRAS, 443, 299

Marscher, A. P. 1980, ApJ, 235, 386

Mészáros, P. 2006, Reports on Progress in Physics, 69, 2259
Mészáros, P., Ramirez-Ruiz, E., Rees, M. J., \& Zhang, B. 2002, ApJ, 578, 812

Meszaros, P. \& Rees, M. J. 2014, ArXiv e-prints

Miller-Jones, J. C. A., McCormick, D. G., Fender, R. P., Spencer, R. E., Muxlow, T. W. B., \& Pooley, G. G. 2005, MNRAS, 363, 867

Mimica, P. \& Aloy, M. A. 2010, MNRAS, 401, 525

Nakar, E. \& Piran, T. 2002, ApJ, 572, L139

Norris, J. P., Nemiroff, R. J., Bonnell, J. T., Scargle, J. D., Kouveliotou, C., Paciesas, W. S., Meegan, C. A., \& Fishman, G. J. 1996, ApJ, 459, 393

Panaitescu, A., Spada, M., \& Mészáros, P. 1999, ApJ, 522, L105

Pe'er, A. 2008, ApJ, 682, 463

—. 2014, Space Sci. Rev., 183, 371

-. 2015, Advances in Astronomy, 2015, 907321

Piran, T. 2004, Reviews of Modern Physics, 76, 1143

Ramirez-Ruiz, E. \& Fenimore, E. E. 2000, ApJ, 539, 712

Rees, M. J. 1978, MNRAS, 184, 61P

Rees, M. J. \& Meszaros, P. 1994, ApJ, 430, L93

Rybicki, G. B. \& Lightman, A. P. 1979, Radiative processes in astrophysics

Ryde, F. 2004, ApJ, 614, 827

-. 2005, ApJ, 625, L95

Ryde, F. \& Pe'er, A. 2009, ApJ, 702, 1211

Ryde, F., Pe'er, A., Nymark, T., et. al. 2011, MNRAS, 415, 3693

Sari, R. \& Piran, T. 1995, ApJ, 455, L143

-. 1997, ApJ, 485, 270

Service, A. T. 1986, ApJ, 307, 60

Sikora, M., Begelman, M. C., \& Rees, M. J. 1994, ApJ, 421, 153

Spada, M., Ghisellini, G., Lazzati, D., \& Celotti, A. 2001, MNRAS, 325, 1559

Thompson, C. 1994, MNRAS, 270, 480

Usov, V. V. 1992, Nature, 357, 472

Wang, F. Y. \& Cheng, K. S. 2012, MNRAS, 421, 908

Wiersma, J. 2007, PhD thesis, Utrecht University

Woosley, S. E. \& Bloom, J. S. 2006, ARA\&A, 44, 507

Zhang, B. 2007, Chinese J. Astron. Astrophys., 7, 1

- 2014, International Journal of Modern Physics D, 23, 30002

Zhang, B. \& Mészáros, P. 2002, ApJ, 566, 712 